"C2020 IEEE. Personal use of this material is permitted. Permission from IEEE must be obtained for all other uses, in any current or future media, including reprinting/republishing this material for advertising or promotional purposes, creating new collective works, for resale or redistribution to servers or lists, or reuse of any copyrighted component of this work in other works." 


\title{
Low-Cost Ultra-Wideband High-Gain Compact Resonant Cavity Antenna
}

\author{
Touseef Hayat, Student Member, IEEE, Muhammad U. Afzal, Member, IEEE, Foez Ahmed, Student \\ Member, IEEE, Shiyu Zhang, Member, IEEE, Karu P. Esselle, Fellow Member, IEEE, Yiannis \\ Vardaxoglou, Fellow Member, IEEE
}

\begin{abstract}
A 3D printed planar permittivity-gradient superstrate (PGS) is used to improve the directive radiation characteristics of a waveguide fed compact resonant cavity antenna (C-RCA). Farfield directivity of classical uniform superstrate based RCAs is limited due to non-uniform aperture phase distribution caused by even transmission through the superstrate. Transverse PGS has been used here to remarkably improve aperture phase distribution and hence directive radiation performance of RCAs. Furthermore, the PGS was rapidly prototyped in one hour and 43 minutes using a low cost acrylo-butadiene styrene (ABS) filament without using traditional multistep milling and machining. Single step fabrication was performed and effective dielectric constant of the ABS was varied through controlled infill percentage in different regions of the PGS. Measurements of a prototype indicate unrivaled results, from a smaller footprint, which includes peak directivity of $16.048 \mathrm{~dB}, 3 \mathrm{~dB}$ directivity bandwidth of $49.65 \%$ and side lobe levels lower than $\mathbf{- 1 0 . 4} \mathrm{dB}$ throughout the operating frequency band. The 3D printed PGS thus outperforms all previously reported superstrates, for RCAs, by demonstrating similar radiation performance with an equivalent material cost of only 0.41 USD.
\end{abstract}

Index Terms - 3D printing, additive manufacturing, directivity bandwidth, directivity enhancement, acrylo-butadiene styrene (ABS), radiation characteristics, resonant cavity antenna (RCA), rapid prototyping.

\section{INTRODUCTION}

C avity-backed resonator antennas or resonant cavity antennas (RCAs) have drawn an unprecedented attention in recent years because they offer directive radiation characteristics, compared to traditional antennas, with simple configuration and non-complex feeding mechanism [1], [2]. These antennas are also referred in literature as Fabry-Perot antennas, partially reflecting surface antennas, and electromagnetic band resonator antennas. The core of RCAs is a resonant cavity that is formed between a reflecting superstrate suspended on top of an electromagnetic source, at an appropriate distance. The cavity resonates at the operating frequency thus resulting in enhanced directional radiation.

This concept was first proposed and demonstrated by Trentini in 1956 [3]. One of major challenges with RCAs was their narrowband impedance matching and limited directivity bandwidth [4], [5]. In 1990, few numerical techniques like

T. Hayat and F. Ahmed are with the School of Engineering, Macquarie University, Sydney, Australia, (Email:touseef.hayat@students.mq.edu.au).

M. Afzal and K.P. Esselle are with the School of Engineering and Data Science, University of Technology Sydney, Australia.

S. Zhang and Y. Vardaxoglou are with the Wolfson School of Mechanical,

Electrical and Manufacturing Engineering, Loughborough University, UK.

This work has been funded by International Macquarie University Research Excellence Scholarship (iMQRES). integral equations, partial differential equations and method of moments were implemented to optimize performance of superstrate loaded RCAs, which enhanced the directivity bandwidth to $9-15 \%$ [6], [7]. During the last decade, highly reflective FSS type superstrates, having sub-wavelength thicknesses, were used to achieve similar bandwidth [8]. Nevertheless, bandwidth remained one of the biggest challenges with the RCAs until the development of compact resonant cavity antennas (C-RCA) having unprinted or alldielectric type permittivity-gradient superstrates (PGSs). CRCAs have 3dB gain bandwidth between 50-60\% [1], [9] and peak gain between 15 to $20 \mathrm{dBi}$, which is sufficient for medium gain antennas and arrays of these antennas can be used for highgain antenna applications.

Despite exceptional radiation performance, high-cost of superstrates having multiple commercial dielectric materials prohibitively restricts the wide-scale use of the C-RCAs. The proposed design addresses this limitation by demonstrating a new type of a C-RCA that makes use of a low-cost and light weight 3D printed plastic superstrate. The superstrate has an effective gradient in dielectric material, similar to the earlier reported C-RCA [2], [10]. Instead of using several dielectric materials, the proposed superstrate design achieves effective permittivity gradient using multiple processes having different infill percentages, of a same dielectric filament, in circular regions of the superstrate. The plastic material used for the 3D printing is readily available acrylo-butadiene styrene (ABS) filament. The proposed C-RCA has measured $3 \mathrm{~dB}$ directivity bandwidth of $\simeq 50 \%$ which is comparable to any of the earlier reported expensive $\mathrm{C}$-RCAs.

The rest of the letter has been organized in five sections

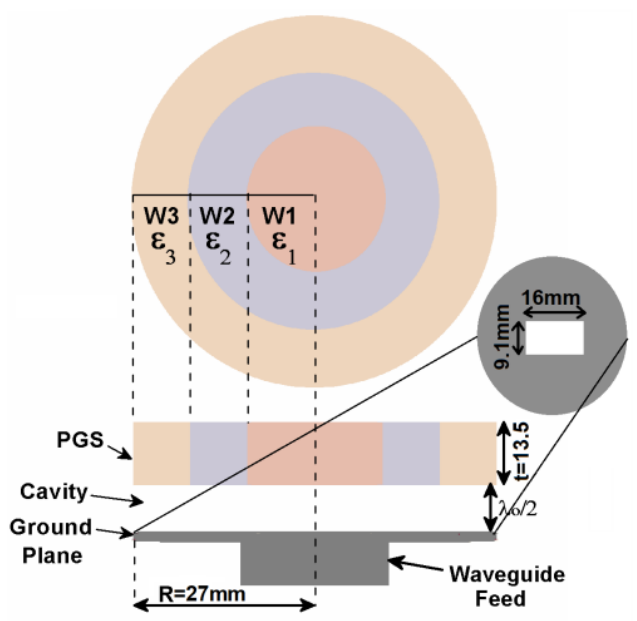

Fig. 1: Configuration of cavity antenna formed with PGS, top and side views of superstrate shown. 
including an introduction and a conclusion sections. In Section II, we will explain configuration to give physical insight into the non-uniform superstrate based RCAs. Section III explains synthesis and prototyping of non-uniform superstrate designed by varying the infill percentage of material during $3 \mathrm{D}$ printing process. While predicted and measured results are presented in Section IV.

\section{Configuration of Permittivity-Gradient SUPERSTRATE (PGS)}

Classical RCAs comprise a primary feed with perfect electric conductor ground plane and a uniform partially reflecting superstrate. The ground plane and the superstrate are separated by a half-wavelength $\left(\lambda_{0} / 2\right)$ space, where $\lambda_{0}$ is the free-space wavelength at the operating frequency. This air-filled region between the ground plane and the superstrate forms a cavity, which is excited by primary feed and resonates at the operating frequency. The classical RCAs made from uniform superstrates are known to have non-uniform aperture phase distribution outside the cavity, particularly in a wider frequency band [11]. The uniformity in aperture phase distribution is enhanced by using superstrates having laterally varying transmission phase delays - more precisely decreasing phase delays - from the center towards edges [2]. This work focuses developing extremely low-cost varying phase delay superstrates using readily available plastic materials and $3 \mathrm{D}$ printers.

It is well known, that the phase delay of an electromagnetic field propagating through dielectric can be varied either using dielectric materials with same thickness and different permittivity values or using single dielectric and varying its thickness. The permittivity variation is of great interest because it leads to planar geometry that eliminates drawbacks such as aperture shadowing associated with the non-planar superstrates [12]. Keeping this in mind and taking inspiration from earlier reported work on C-RCAs [1], [2], [13], we focused on permittivity-gradient type superstrates. A superstrate was developed having three concentric rings of electrically different dielectric regions. The number of concentric rings were set to three because excessively increasing the number of rings does not influence the performance significantly, rather it makes the fabrication complex [2]. The superstrate is suspended at a spacing of $13.7 \mathrm{~mm}\left(\approx \lambda_{0} / 2\right)$ over ground plane, which is condition for ideal resonance of C-RCA to attain optimal radiation performance [14], [15]. The ground is a metal sheet of $27 \mathrm{~mm}$ radius, which has a feeding slot $(16 \mathrm{~mm} \times 9.1 \mathrm{~mm})$ in the middle, for WR-75 waveguide adapter, as shown in Fig. 1. The widths of three regions (from center to outer edges) of the superstrate and their corresponding dielectric constants are selected through parametric study, which for brevity is not presented here but details on working can be found in earlier publications on C-RCAs [2], [10]. The optimal parameters values are $\mathrm{w}_{1}=10 \mathrm{~mm}, \mathrm{w}_{2}=\mathrm{w}_{3}=8.5 \mathrm{~mm}, \varepsilon_{1}=2.8 \varepsilon_{2}=2.4$ and $\varepsilon_{3}=2.1$. The thickness of the all three dielectrics in superstrate is 13.5 $\mathrm{mm}$. The aperture size of the superstrate and width of concentric regions can be altered as they depend upon aperture size. In this design, we considered the circular aperture of size $0.95 \lambda_{\mathrm{L}}(28.3$ $\mathrm{mm})$, where, $\lambda_{\mathrm{L}}$ is free space wavelength at the lowest operating frequency $(10.6 \mathrm{GHz})$.

The superstrate was developed using low cost and low-loss
ABS filament, which has dielectric constant of 2.8 [16]. It is to be mentioned here that by varying the infill percentage during the $3 \mathrm{D}$ printing, it is only possible to effectively achieve dielectric constant value less than the value of the plastic material. So for design of C-RCA, the use of polylactic acid (PLA) and acrylonitrile styrene acrylate (ASA) filaments will be equally effective as they have respective relative permittivity of 2.72 and 3.4, which is very close to ABS [17]. But ABS material is much easier to print and is more robust compared to other aforementioned filaments. Further details on the effect of infill percentage on effective permittivity values are given in the following section.

\section{EFFEctive Permittivity AND SuPERSTRATE PROTOTYPING}

A fundamental parameter that controls the density of a $3 \mathrm{D}$ printed material and hence its electrical properties is the infill percentage [18]. Apart from the density of the printed object, the infill percentage also affects the mechanical integrity of a printed sample, which may be critical for some applications involving high level of stress. It has been reported previously that there is a linear relation between infill percentage and effective relative permittivity of the 3D printed sample [19]:

$$
v_{i}=\frac{\varepsilon_{r e f f}-1}{\varepsilon_{r}-1}
$$

where, $v_{i}$ is infill percentage between 0 and $1, \varepsilon_{\text {reff }}$ is effective relative permittivity of the printed sample and $\varepsilon_{r}$ is the dielectric constant of the plastic material used for the $3 \mathrm{D}$ printing. The equation can be used to make material of customized effective permittivity by appropriately using the $v_{i}$. As an example, consider the ABS material that has $\varepsilon_{\mathrm{r}}=2.8$. In any $3 \mathrm{D}$ printed sample, by varying $v_{i}$ between 0 and 1 , an effective permittivity value between 1 and 2.8 can be achieved. To validate this, we printed five rectangular samples with dimensions equivalent to WR-90 having arbitrary infill percentages of $100 \%, 85 \%, 70 \%$, $40 \%$ and $20 \%$. Waveguide setup measurement was performed to record complex reflection and transmission coefficients of the samples using E4991A impedance analyzer in the X-band. Effective dielectric constants and loss tangents were calculated using Nicholas-Ross Weir (NRW) method as explained in [20]. The plot of measured effective permittivity and loss tangents for all five samples and air (for reference) are given in Fig. 2.

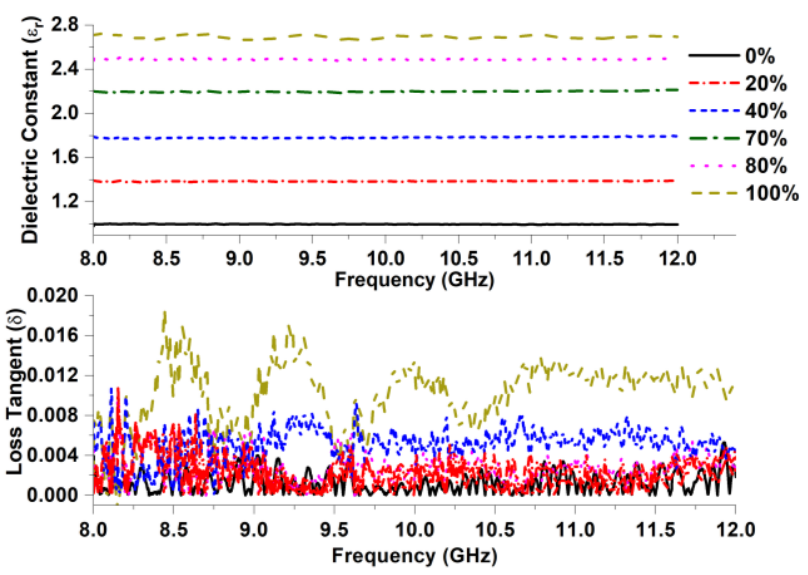

Fig. 2: Measured dielectric constant and loss tangent for 3D printed samples with different infill percentages. 


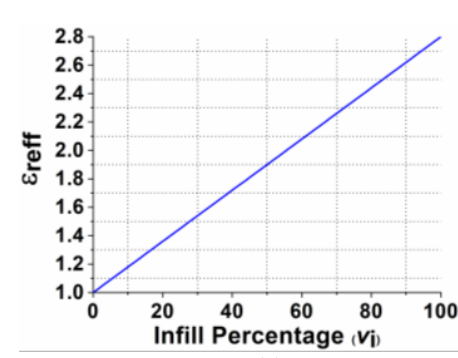

(a)

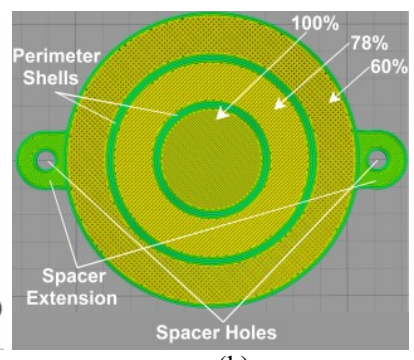

(b)
Fig. 3: (a) Variation in effective dielectric constant with change in infill percentage for ABS (b) Screenshot of preview mode of simplify3D software during print setup.

The theoretical value of the effective permittivity is calculated using (1), by varying the infill percentage and are plotted in Fig. 3(a). The measured values of the five samples are in good agreement with the theoretical values. This ability to control effective relative permittivity of material using infill percentage of 3D printed material can be considered as a leap forward in the design of C-RCAs because with traditional machining methods only few discrete commercially available dielectrics can be used.

Using (1) and the plot in Fig. 3(a), we selected the infill percentages of $v_{i}=100 \%, 78 \%$ and $60 \%$ for the three dielectric constants of 2.8, 2.4 and 2.1, respectively, required for the PGS. Three rings of PGS simulation file were exported as separate Standard Tessellation Language (.stl) files from CST studio Suite. The '.stl' files were imported in Simplify3D software and separate processes were added against each file with their corresponding infill percentages. Two solid outline/perimeter shells were introduced at the edge of each ring to join all the stl's. The shells were not only useful to integrate the three rings but also enhance the robustness of the structure. Additionally, two extensions were introduced at opposite ends of the last ring to physically fix the PGS, with M3 screws and spacers, above the waveguide feed.

To 3D print the superstrate rings, layer height was set to $0.2 \mathrm{~mm}$, extruder temperature was fixed to $240^{\circ} \mathrm{C}$ and bed temperature was set to $110^{\circ} \mathrm{C}$. With these settings, simplify3D estimated the build time of 1 hour and 43 minutes, consuming $8.195 \mathrm{~m}$ of ABS filament, with equivalent material weight and cost of $16.4 \mathrm{~g}$ and $0.41 \mathrm{USD}$, respectively. Cross-sectional view screenshot captured during the print setup indicating different

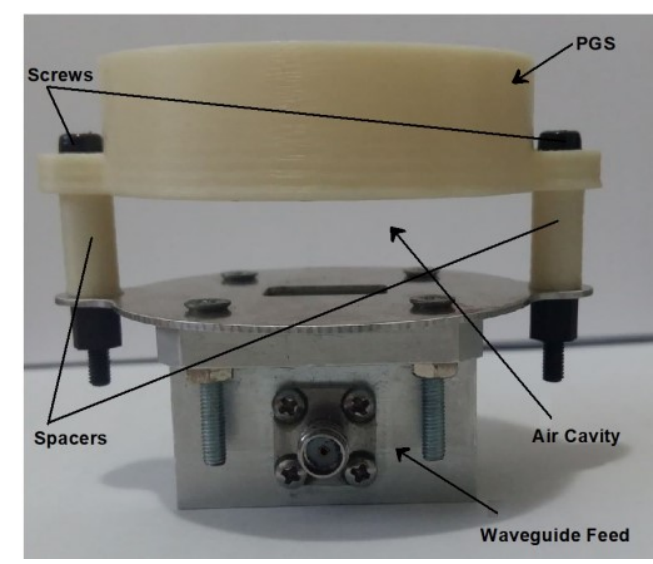

Fig. 4: Photograph of 3D printed PGS prototype with RCA

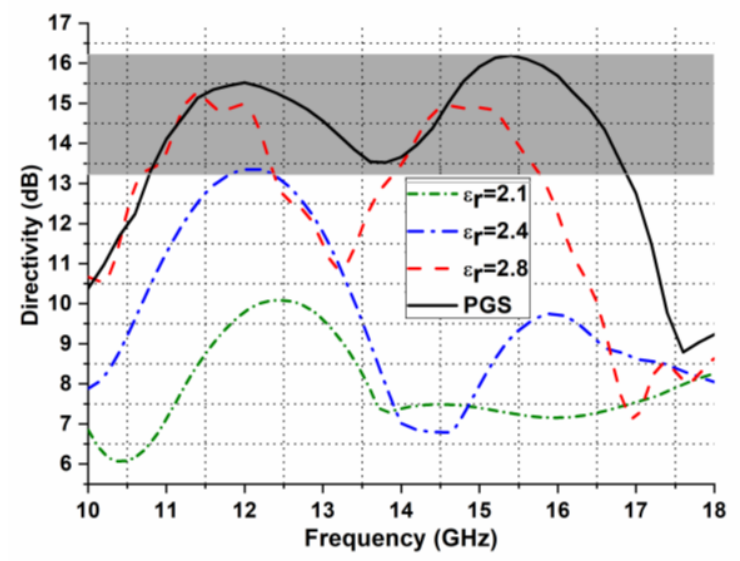

Fig. 5: Directivity comparison for PGS and homogenous dielectric superstrates.

regions of the PGS is presented in Fig. 3(b). The final factory file was converted to G-code and Omni3D printer was used to print the prototype in single step. A picture of the printed superstrate with waveguide feed is shown in Fig. 4.

\section{Measurement and Results}

The 3D model of the C-RCA was simulated with the CST Microwave Studio. To highlight the superior performance of the PGS based C-RCA, we also simulated and compared three RCAs having uniform dielectric superstrates of different permittivity values but the same aperture size as that of C-RCA. Comparison between the variations of peak directivity in desired frequency band for uniform superstrates and PGS based RCAs is shown in Fig. 5. It can be noted that for uniform superstrates, maximum peak directivity is higher for the RCAs with higher relative permittivity of the superstrate, however, this results in decreasing the $3 \mathrm{~dB}$ directivity bandwidth. Using PGS, maximum peak directivity is higher than that attained with any of the dielectric superstrates. Meanwhile, the $3 \mathrm{~dB}$ directivity bandwidth with the PGS increases by $20.32 \%$. This is because of tremendous improvement in near-field aperture

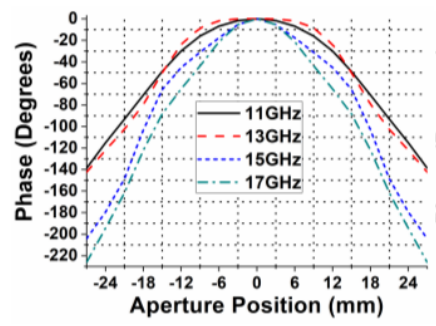

(a)

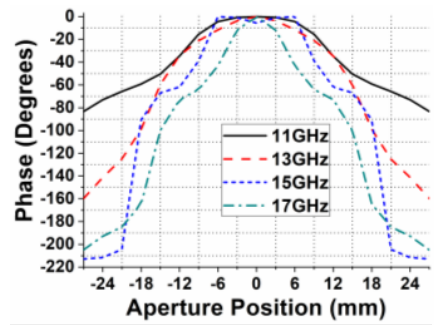

(c)

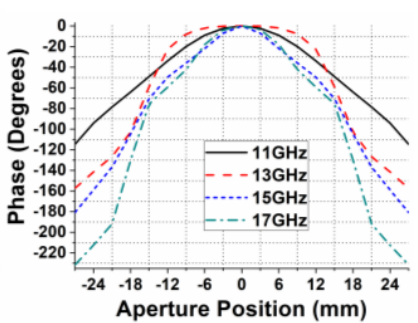

(b)

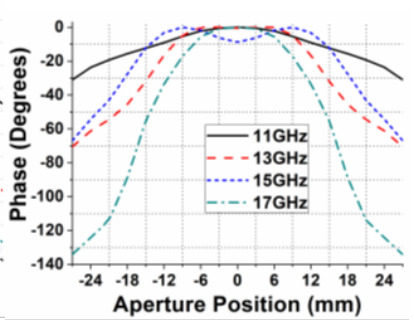

(d)
Fig. 6: E-Field distribution on aperture at four distinct frequencies within operating band (a) $\varepsilon_{\mathrm{r}}=2.1$ (b) $\varepsilon_{\mathrm{r}}=2.4$ (c) $\varepsilon_{\mathrm{r}}=2.8$ and (d) PGS. 
TABLE I: PERFORMANCE COMPARISON OF PGS WITH UNIFORM SUPERSTRATES.

\begin{tabular}{|c|c|c|c|c|c|c|}
\hline Dielectric & Peak & 3dB Dir. & \multicolumn{4}{|c|}{ Maximum phase variation } \\
\cline { 4 - 7 } $\begin{array}{c}\text { constant } \\
\left(\varepsilon_{\mathrm{r}}\right)\end{array}$ & $\begin{array}{c}\text { Dir. } \\
(\mathrm{dB})\end{array}$ & $\begin{array}{c}\text { Bandwidth } \\
(\%)\end{array}$ & $\begin{array}{c}11 \mathrm{GHz} \\
(\text { deg. })\end{array}$ & $\begin{array}{c}13 \mathrm{GHz} \\
(\text { deg. })\end{array}$ & $\begin{array}{c}15 \mathrm{GHz} \\
(\text { deg. })\end{array}$ & $\begin{array}{c}17 \mathrm{GHz} \\
(\text { deg. })\end{array}$ \\
\hline 2.8 & 15.2 & 19.82 & 83.3 & 159.8 & 204.9 & 212.9 \\
\hline 2.4 & 13.3 & 21.38 & 114.5 & 157.3 & 180.8 & 231.5 \\
\hline 2.1 & 10.0 & 19.33 & 138.7 & 142.4 & 204.3 & 226.1 \\
\hline PGS & 16.2 & 49.65 & 30.9 & 70.47 & 66.9 & 134.1 \\
\hline
\end{tabular}

phase distribution with the PGS. For the comparison we probed the electric field at a distance of $0.5 \mathrm{~mm}$ above the C-RCA and aperture plane. The maximum phase variation is simply calculated by taking difference between highest and lowest phase values in the probed phase data. It is an indicative figure of non-uniformity in the aperture phase distribution that helps to predict far-field directive radiation characteristics of an antenna. Phase of the dominant radiating electric field component, taken at four different frequencies within the operating band is shown in Fig. 6(a)-(d). From the figure, it can be comprehended that for C-RCAs with uniform superstrates, the phase variation is smallest at $11 \mathrm{GHz}$ and it is largest at 17 GHz. With the PGS, the phase is nearly uniform at $11 \mathrm{GHz}$, having smallest phase variation of $30^{\circ}$ from center to the edge, and largest phase variation of about $130^{\circ}$ at $17 \mathrm{GHz}$. A summary of performance comparison between uniform superstrates and PGS in terms of peak directivity, $3 \mathrm{~dB}$ directivity bandwidth and aperture phase distribution is given in Table. I. The plot of input reflection coefficient is shown in Fig. 7, which shows that reflection magnitude is less than -10 $\mathrm{dB}$ in the frequency range between $9 \mathrm{Ghz}$ to $20 \mathrm{GHz}$.

The far-field radiation measurements were performed in NSI Spherical Near-Field chamber. The measured prototype has $3 \mathrm{~dB}$ directivity bandwidth of $49.65 \%$ (10.6 to $17.6 \mathrm{GHz}$ ) along with the peak directivity of $16.1 \mathrm{dBi}$. Measured peak directivity with varying frequency is plotted in Fig. 8. Measured realized gain is also included in the same figure in a frequency band between $10 \mathrm{GHz}$ and $15 \mathrm{GHz}$. Gain was calculated using gain by comparison method and was not measured for full band because of the unavailability of standard gain horn (SGH) in the measurement facility for the frequency values beyond $15 \mathrm{GHz}$.

The far-field elevation pattern cuts along H-Plane at two different frequencies are given in Fig. 9. The side-lobe levels are below -12.7 at all frequency points. To highlight the effectivity of this work, the Table II compares the directive

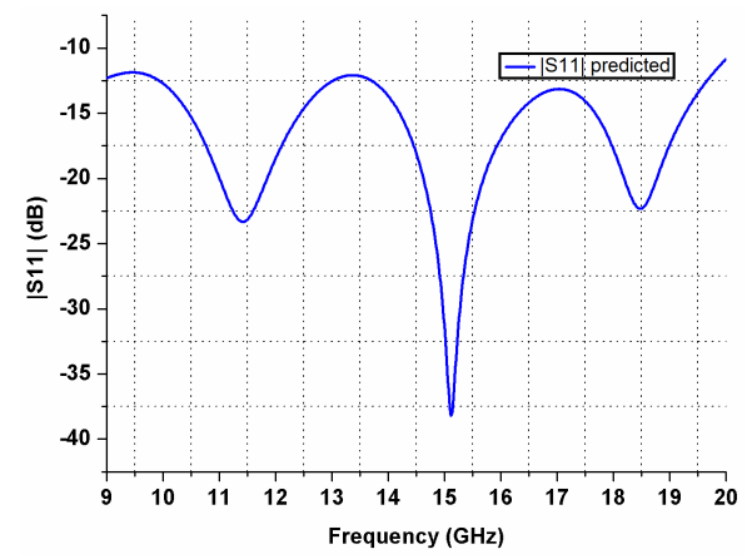

Fig. 7. Predicted and measured input reflection of C-RCA.

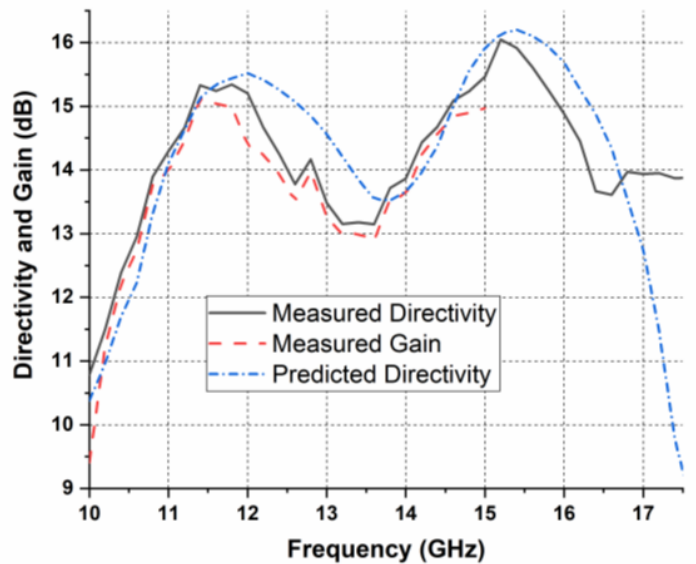

Fig. 8: Predicted and measured directivity and gain.

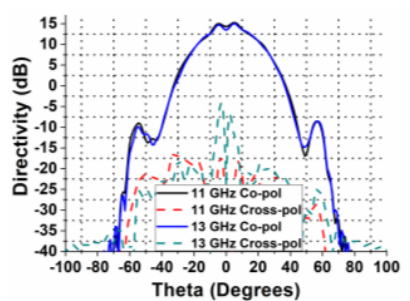

(a) (b)

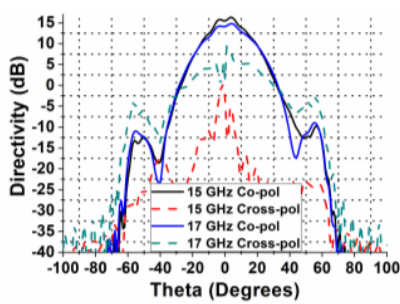

Fig. 9: Measured co- and cross-polarized components of C-RCA at: (a) 11 and $13 \mathrm{GHz}$. (b) 15 and $17 \mathrm{GHz}$.

TABLE II: COMPARISON OF RCA WITH OTHER SUPERSTRATES.

\begin{tabular}{|c|c|c|c|c|c|c|c|}
\hline Ref. & $\begin{array}{c}\text { Peak Dir } \\
(\mathrm{dB})\end{array}$ & $\begin{array}{c}3 \mathrm{~dB} \text { Dir } \\
\text { bw. }\end{array}$ & $\begin{array}{c}\text { SLLs } \\
(\mathrm{dB})\end{array}$ & Cost & Area $\left(\lambda_{\mathrm{L}}{ }^{2}\right)$ & DBP & DBP/A \\
\hline$[1]$ & 20.7 & $55.0 \%$ & -10 & High & 14.94 & 6462 & 432 \\
\hline$[2]$ & 16.6 & $54.7 \%$ & -10 & High & 1.54 & 2500 & 1623.3 \\
\hline$[10]$ & 20.7 & $56.0 \%$ & -12 & High & 5.3 & 6662 & 1257 \\
\hline This work & 16.05 & $49.65 \%$ & -10.4 & Low & 2.86 & 1999 & 699 \\
\hline
\end{tabular}

radiation performance of propsed PGS with some of the most recently published superstrates based RCAs. The C-RCA has matching performance with its counterparts in terms of directivity, side lobe levels (SLLs), directivity bandwidth product (DBP) and DBP per unit Area (DBP/A). While, it is developed at only fractional cost to that required to prototype previously reported RCAs, which mostly use commerically avaialble dielectric substrates and delicate manufacturing processes.

\section{CONCLUSION}

Additively manufactured permittivity-gradient superstrate (PGS) with small footprint is used with a primary feed to develop a low-cost wideband compact resonant cavity antenna (C-RCA). The C-RCA radiates planar phase fronts having negligible aperture phase variation in a wide frequency band. A prototype is developed by varying the infill percentage of an ABS dielectric material within different annular rings of the PGS. Using the Omni 3D printer, the fabrication of PGS takes 1 hour and 43 minutes with consumption of $15.6 \mathrm{~g}$ of ABS filament. The PGS is extremely cost effective and can be rapidly prototyped to realize $\mathrm{C}-\mathrm{RCAs}$ having similar radiation performance as that reported for other RCAs. 


\section{REFERENCES}

[1] A. P. Feresidis and J. C. Vardaxoglou, "A broadband high-gain resonant cavity antenna with single feed," in 2006 First European Conference on Antennas and Propagation, 2006, pp. 1-5.

[2] R. M. Hashmi and K. P. Esselle, "A Class of Extremely Wideband Resonant Cavity Antennas With Large Directivity-Bandwidth Products," IEEE Trans. Antennas Propag., vol. 64, no. 2, pp. 830835, Feb. 2016.

[3] G. V. Trentini, "Partially reflecting sheet arrays," IRE Trans. Antennas Propag., vol. 4, no. 4, pp. 666-671, Oct. 1956.

[4] O. M. Ramahi and Y. T. Lo, "Superstrate effect on the resonant frequency of microstrip antennas," Microw. Opt. Technol. Lett., vol. 5, no. 6, pp. 254-257, Jun. 1992.

[5] A. Bhattacharyya and T. Tralman, "Effects of dielectric superstrate on patch antennas," Electron. Lett., vol. 24, no. 6, p. 356, 1988.

[6] F. Zavosh and J. T. Aberle, "Design of High Gain Microstrip Antennas," Microw. J., vol. 42, no. 9, pp. 138-138, Sep. 2000.

[7] F. Zavosh and J. Aberle, Microwave journal., vol. 42, no. 9. Horizon House-Microwave, 1999.

[8] A. Foroozesh and L. Shafai, "Investigation Into the Effects of the Patch-Type FSS Superstrate on the High-Gain Cavity Resonance Antenna Design," IEEE Trans. Antennas Propag., vol. 58, no. 2, pp. 258-270, Feb. 2010.

[9] R. M. Hashmi and K. P. Esselle, "A Class of Extremely Wideband Resonant Cavity Antennas With Large Directivity-Bandwidth Products," IEEE Trans. Antennas Propag., vol. 64, no. 2, pp. 830835, Feb. 2016.

[10] A. A. Baba, R. M. Hashmi, and K. P. Esselle, "Achieving a Large Gain-Bandwidth Product From a Compact Antenna," IEEE Trans. Antennas Propag., vol. 65, no. 7, pp. 3437-3446, Jul. 2017.

[11] M. U. Afzal, K. P. Esselle, and B. A. Zeb, "Dielectric PhaseCorrecting Structures for Electromagnetic Band Gap Resonator Antennas," IEEE Trans. Antennas Propag., vol. 63, no. 8, pp. 33903399, Aug. 2015.

[12] A. Petosa and A. Ittipiboon, "Shadow blockage effects on the aperture efficiency of dielectric Fresnel lenses," IEE Proc. Microwaves, Antennas Propag., vol. 147, no. 6, p. 451, 2000.

[13] M. A. Al-Tarifi, D. E. Anagnostou, A. K. Amert, and K. W. Whites, "The puck antenna: A compact design with wideband, high-gain operation," IEEE Trans. Antennas Propag., vol. 63, no. 4, pp. 1868 1873, Apr. 2015.

[14] Y. Ge, K. P. Esselle, and T. S. Bird, “A Method to Design Dual-Band, High-Directivity EBG Resonator Antennas Using Single-Resonant, Single-Layer Partially Reflective Surfaces," Prog. Electromagn. Res. C, vol. 13, pp. 245-257, 2010.

[15] S. Vaid and A. Mittal, "Wideband Orthogonally Polarized Resonant Cavity Antenna with Dual Layer Jerusalem Cross Partially Reflective Surface," Prog. Electromagn. Res. C, vol. 72, pp. 105-113, 2017.

[16] T. Hayat, M. U. Afzal, A. Lalbakhsh, and K. P. Esselle, "Additively Manufactured Perforated Superstrate to Improve Directive Radiation Characteristics of Electromagnetic Source," IEEE Access, pp. 1-1, 2019.

[17] T. Hayat, M. U. Afzal, A. Lalbakhsh, and K. P. Esselle, "3-D-Printed Phase-Rectifying Transparent Superstrate for Resonant-Cavity Antenna," IEEE Antennas Wirel. Propag. Lett., vol. 18, no. 7, pp. 1400-1404, Jul. 2019.

[18] E. Massoni et al., "Characterization of 3D-printed dielectric substrates with different infill for microwave applications," 2016 IEEE MTT-S International Microwave Workshop Series on Advanced Materials and Processes for RF and THz Applications (IMWS-AMP), Chengdu, 2016, pp. 1-4.

[19] S. Zhang, R. K. Arya, S. Pandey, Y. Vardaxoglou, W. Whittow, and R. Mittra, "3D-printed planar graded index lenses," IET Microwaves, Antennas Propag., vol. 10, no. 13, pp. 1411-1419, Oct. 2016.

[20] A. M. Nicolson and G. F. Ross, "Measurement of the Intrinsic Properties Of Materials by Time-Domain Techniques," IEEE Trans. Instrum. Meas., vol. 19, no. 4, pp. 377-382, 1970. 